\title{
Medical Pathologies and Hut Guardians' Ability to Provide First Aid in Mountain Huts: A Prospective Observational Study
}

Marc Blancher, MD; Jérôme Colonna d'Istria, MD; Amandine Coste, MD; Philippine Saint Guilhem, MD; Antoine Pierre, MD; Flora Clausier, MD; Guillaume Debaty, MD, PhD; Jean Luc Bosson, MD, PhD; Raphaël Briot, MD, PhD; Pierre Bouzat, MD, PhD

From the Département de médecine d'urgence, Centre Hospitalier Universitaire (CHU) de Grenoble-Alpes, Grenoble, France (Drs Blancher, Saint Guilhem, Debaty, and Briot); the Association Nationale des Médecins et Sauveteurs en Montagne, (ANMSM) French Mountain Rescue Association (Drs Blancher, Colonna d'Istria, and Briot); the Département de médecine générale, Université Joseph Fourrier, Faculté de médecine, Grenoble, France (Drs Coste, Pierre, and Clausier); the Centre d'Investigation Clinique, Grenoble, France (Dr Bosson); the Pôle Anesthésie Réanimation, Centre Hospitalier Universitaire de Grenoble, Grenoble, France (Dr Bouzat); the Grenoble Institut des Neurosciences, Grenoble, France (Dr Bouzat); and the Grenoble Alpes Université, Grenoble, France (Drs Bosson, Bouzat, and Briot).

Objective.-To describe the resources for medical condition management in mountain huts and the epidemiology of such events.

Methods.-We conducted a 3-step study from April 2013 to August 2014 in French mountain huts. The first step consisted of collecting data regarding the first aid equipment available in mountain huts. The second step consisted of a qualitative evaluation of the mountain hut guardian's role in medical situations through semistructured interviews. Finally, a prospective observational study was conducted in the summer season to collect all medical events (MEs) that occurred during that period.

Results.-Out of 164 hut guardians, $141(86 \%)$ had a basic life support diploma. An automatic external defibrillator was available in $41(26 \%)$ huts, and 148 huts $(98 \%)$ were equipped with a first aid kit. According to semistructured interviews, hut guardians played a valuable role in first aid assistance. Regarding the observational study, 306 people requested the hut guardian's help for medical reasons in 87 of the 126 huts included. A total of 501 MEs for approximately 56,000 hikers (0.85\%) were reported, with 280 MEs (56\%) involving medical pathologies and 221 (44\%) MEs involving trauma-related injuries.

Conclusions.-MEs had low prevalence, but the hut guardian played a valuable role as a first aid responder.

Key words: mountain hut, hut guardian, wilderness medicine, mountain emergency medicine, first aid

\section{Introduction}

In European countries, hikers, climbers, and alpinists use huts for accommodation in remote mountainous areas. Hut guardians assist people with food and accommodation and may provide advice. When a medical situation occurs close to a hut, hut guardians are often considered the first resource for providing initial assistance, which may consist of contacting mountain rescue services to manage a helicopter evacuation.

Corresponding author: Dr. Marc Blancher, Département de médecine d'urgence, Centre Hospitalier Universitaire (CHU) de Grenoble-Alpes, F-38043, Grenoble, France (e-mail: MBlancher@chu-grenoble.fr).

Submitted for publication April 2016.

Accepted for publication August 2016.
However, first aid assistance to mountaineers is not standardized in mountain huts. Twenty years ago, the International Commission for Alpine Rescue (ICAR) detailed the content of a hut's first aid kit. ${ }^{1}$ More recently, this commission also recommended the implementation of an automated external defibrillator (AED) in mountain huts. ${ }^{2}$ In France, the association Pharefuge assisted more than 90 mountain huts with first aid training and kits for hut guardians, but a precise inventory of these resources is not available. Moreover, the exact epidemiology of medical events (MEs) occurring in mountain huts remains unknown; improving our knowledge on this topic would help in adjusting the information and contents of first aid kits. The aims of this study were 1) to describe the actual first 
aid resources available in French mountain huts; 2) to define hut guardians' role according to professionals involved in hut keeping and mountain rescue; and 3) to establish the epidemiology of medical pathologies occurring in the vicinity of mountain huts.

\section{Methods}

We conducted a 3-step study from April 2013 to August 2014 in the 236 French mountain huts. The first step consisted of collecting data regarding the actual first aid equipment in mountain huts through a dedicated questionnaire from April to June 2013. The second step consisted of a qualitative evaluation of the mountain hut guardian's role in medical situations through semistructured interviews of hut guardians and healthcare professionals (June-September 2013). Finally, a prospective observational study was conducted from July 2014 to August 2014 to collect all MEs that occurred within this period and were declared using a dedicated document.

This study was approved by the Research Ethics Board of the French Society of Anesthesiology and Intensive Care (IRB No. 010254) and was also declared to the French National Committee for the protection of public liberties.

Before study completion, we performed a literature search using PubMed, Cochrane, Google Scholar, Medline, and EM Premium databases. The following MeSH terms were used: "mountain huts," "hut keepers," "hut guardian," "mountain rescue," "first aid kit," "acute mountain sickness," "mountaineering," "hiking," "rock climbing," "cardiovascular risk," "mountain wilderness," "alpinism," "first aid knowledge," and "mountain medicine."

\section{INITIAL SURVEY}

All French hut guardians were identified and contacted by phone to explain the purpose of the survey. They received a personalized email, which enabled them to access the survey and complete the online questionnaire. A paper-based questionnaire was mailed to all nonrespondents with a postage-paid return envelope. It consisted of 42 questions divided into 4 domains: 1) characteristics of the mountain hut (location, altitude, specific mountain activities, number of beds); 2) characteristics of the hut guardian (age, gender, experience in hut keeping, and first aid diploma); 3) access to first aid kit; and 4) AED availability.

\section{SEMISTRUCTURED INTERVIEWS}

Two different investigators led individual semistructured interviews of a panel of hut guardians and attendant rescuers and emergency physicians (see online supplementary file 1). The panel was chosen to be representative of different locations, altitudes, and hut management across the Northern French Alps. All participants were recruited by phone, and face-to-face interviews were conducted locally in the mountain hut for hut guardians. Interviews were recorded in full and transcribed anonymously. Transcripts were coded separately using thematic analysis by the 2 investigators. Interviews stopped when no new concept or new idea appeared in 2 successive interviews, corresponding to "data saturation" as defined by Guest et al. ${ }^{3}$ Following the recommendations for explicit and comprehensive report of qualitative studies, the 32-item checklist for interviews and focus groups was used. All items of the Consolidated Criteria for Reporting Qualitative Research (COREQ) list were completed, except for repeat interviews (item no. 18), transcripts returned (item no. 23), and participant checking reporting (item no. 28) due to practical considerations.

\section{PROSPECTIVE OBSERVATIONAL STUDY OF MEDICAL EVENTS IN MOUNTAIN HUTS}

All hut guardians from the survey were contacted to participate in the study. One month before the study period, 30 medical questionnaires were sent to each hut guardian (see online supplemental file 2). Questionnaires were strictly anonymous. Any ME requiring medical assistance by a hut guardian within the study period was included. Hut guardians were responsible for filling out the dedicated document. This document was divided into 5 parts: 1) general considerations (hut's location and activities in the surrounding area); 2) medical symptoms; 3) trauma symptoms (including pain assessment); 4) medical management (basic management, medical care by telemedicine or physician-staffed mountain rescue operation); and 5) evacuation process (if applicable). Questionnaires were returned to the investigators by postage-paid return envelope.

\section{STATISTICAL ANALYSIS}

Descriptive statistics included frequencies and percentages for categorical variables and the mean \pm SD for continuous variables. Independent factors associated with AED availability were provided using a stepwise regression model (Stata version 12.0 software; Statacorp, College Station, TX). Interactions between altitude and huts' attendance were tested. Categories for huts' attendance were divided as follows: $<900$ overnight occupants per hut per year, between 900 and 1499 overnight occupants per hut per year, between 1500 and 2379 overnight occupants per hut per year, and $>2380$ 
overnight occupants per hut per year. Categories for altitude were $<1800 \mathrm{~m}$, between 1800 and $2400 \mathrm{~m}$, and $>2400 \mathrm{~m}$. Odds ratios (OR), and their $95 \%$ CIs were reported. Correlations were assessed using linear regression and Pearson's coefficient. Statistical significance was declared when $P \leq .05$.

\section{Results}

\section{INITIAL SURVEY}

Of the 236 French mountain huts, 164 (69.5\%) hut guardians answered the initial survey. The mean hut altitude was $2065 \pm 520 \mathrm{~m}$. One third of the huts $(\mathrm{n}=$ 46) had more than a 50-bed capacity and 9 huts (5\%) could accommodate more than 100 persons. The average number of overnight occupants per hut per year was 1875 nights (for individual data, see Figure 1).

Hut guardians' age ranged from 22 to 77 years. The majority $(60 \%)$ of guardians were male and had more than 10 years of experience in hut keeping. One hundred forty-one hut guardians (86\%) had a basic life support (BLS) diploma, with $45 \%$ trained within a year and $45 \%$ not trained within the last 3 years. BLS promotes adequate blood circulation in addition to breathing (cardiopulmonary resuscitation, use of an AED) through a clear airway (opening/maintaining the airway). An AED was available in $41(26 \%)$ huts. The factors associated with AED availability were altitude higher than $2400 \mathrm{~m}$ (OR 3.1; 95\% CI 1.1-8.9) and large bed capacity (OR 1.7; 95\% CI 1.15-2.52). One hundred forty-eight huts (98\%) were equipped with a first aid kit. Seventeen huts had a first aid kit with a separate compartment reserved for health care providers (modular

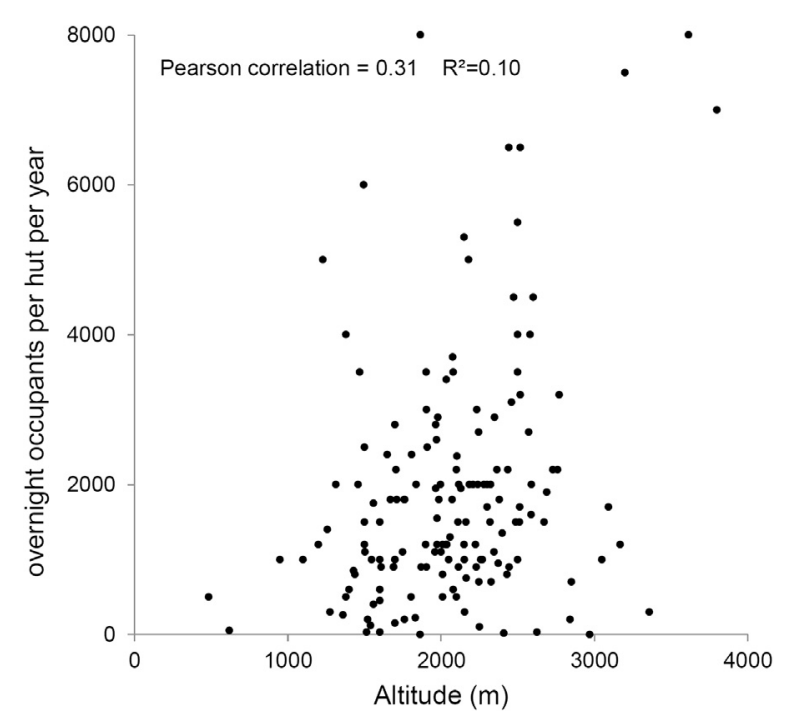

Figure 1. Scatter plot of the overnight occupants per hut per year for each refuge according to its altitude. medical kit). One hundred eight $(73 \%)$ hut guardians participated in first aid kit setup, and maintenance was performed once a year.

\section{SEMISTRUCTURED INTERVIEWS}

Twenty-nine interviews were needed until data saturation was obtained, meaning that no new concept or new idea appeared in 2 successive interviews. Nineteen hut guardians, 7 mountain rescuers, and 6 mountain emergency physicians were interviewed (see online supplementary file 1). The following concepts were identified as relevant (for detailed results, see online supplementary file 1):

1. All of the 29 individuals interviewed considered that hut guardians played a key role in alerting medical mountain rescue services because they could use a specific radio system and could provide information on location and the type of injuries better than the general population.

2. They played a very important role in preventing trauma-related injuries or acute mountain sickness by providing preventive warnings about the ascent.

3. Basic first aid equipment and training adapted to the wilderness environment (BLS) were considered very important. Partnership with a local association (Pharefuge) was highly appreciated for first aid kit management.

4. The hut guardian was considered the key person for mountain rescue organizations and medical dispatching centers in the event of an ME occurring in a hut because 1) information on location and evacuation were better known by the hut guardian, and 2) the hut guardian was a professional and more likely to know whether someone had to be evacuated given the medical situation and geographical considerations. The guardian was also more likely to communicate information to the dispatch center as compared with the general population.

\section{PROSPECTIVE OBSERVATIONAL STUDY REGARDING MEDICAL EVENTS IN MOUNTAIN HUTS}

Of the 164 mountain huts that participated in the initial survey, 126 huts were finally included (Figure 2). Within the study period, 306 people requested the hut guardian's assistance for medical reasons in 87 out of 126 huts included. Thirty-nine huts reported no MEs. A total of 501 MEs were reported (for medical events per hut, arranged by elevation, see Figure 3). Within the study period, the huts included in the study recorded approximately 59,000 mountaineers who spent a night 


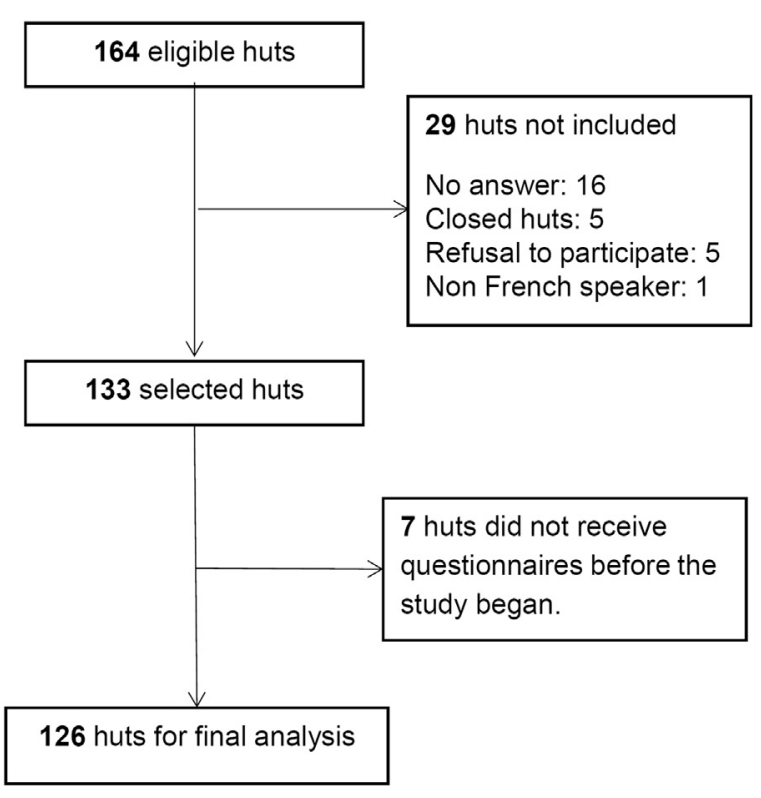

Figure 2. Flow chart of the observational study regarding medical events. The observation lasted 1 season in 126 French mountain huts.

in their accommodations (prevalence of MEs, 0.85\%). A total of 280 MEs (56\%) involved medical pathologies and 221 (44\%) MEs were trauma-related injuries. Details on medical symptoms are given in Table 1. Traumarelated injuries are described in Table 2.

Patients were between 19 and 53 years old; 162 (53\%) patients were men. The most severe cases concerned 4 multiple-trauma patients; 7 patients with chest pain (3 patients with myocardial infarction); 1 cardiac arrest; and 11 cases of acute mountain sickness. Hiking was the most frequent activity correlated with medical issues $(73.5 \%)$, followed by alpinism (15\%) and work-related injuries among hut guardians (7.5\%).

Two hundred seventeen $(71 \%)$ patients used the hut pharmacy. The medications and medical devices used by hut guardians are presented in Figures 4 and 5, respectively. Wound dressing products and paracetamol

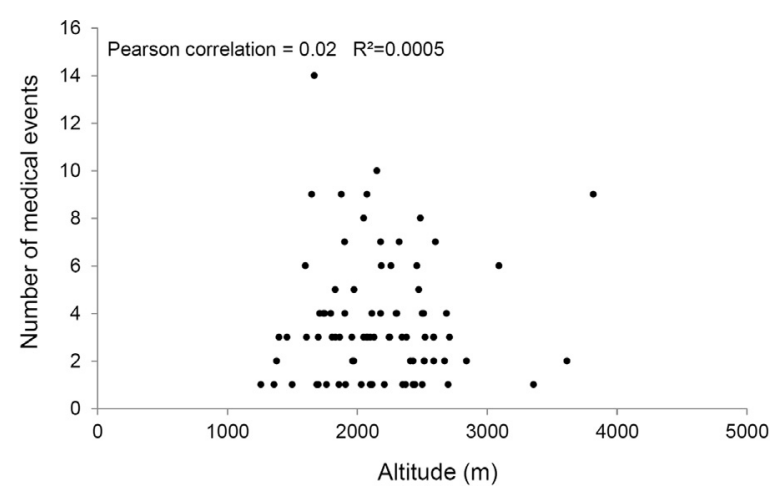

Figure 3. Medical injuries per hut, arranged by elevation (in meters).
Table 1. Characteristics of the 280 medical symptoms occurring in 126 French mountain huts

\begin{tabular}{lc}
\hline Symptoms & $N(\%)$ \\
\hline Headache & $45(16 \%)$ \\
Exhaustion & $36(13 \%)$ \\
Ocular disease & $20(7 \%)$ \\
Nonsevere faintness & $20(7 \%)$ \\
Diarrhea & $18(6 \%)$ \\
Nausea/Vomiting & $18(6 \%)$ \\
Abdominal pain & $16(6 \%)$ \\
Allergic skin disease & $16(6 \%)$ \\
Fever & $14(5 \%)$ \\
Acute mountain sickness & $11(4 \%)$ \\
Dehydration & $9(3 \%)$ \\
Anxiety/Insomnia & $7(3 \%)$ \\
Chest pain & $7(3 \%)$ \\
Dyspnea/Cough & $7(3 \%)$ \\
Dysphagia & $5(2 \%)$ \\
Dizziness & $5(2 \%)$ \\
Common cold & $5(2 \%)$ \\
Urogenital disease & $5(2 \%)$ \\
Heart palpitations & $4(1 \%)$ \\
Moderate hypothermia & $2(1 \%)$ \\
Sun stroke & $2(1 \%)$ \\
Asthma & $2(1 \%)$ \\
Sunburn & $2(1 \%)$ \\
Constipation & $1(0.3 \%)$ \\
Dental disease & $1(0.3 \%)$ \\
Frostbite & $1(0.3 \%)$ \\
Cardiac arrest & $1(0.3 \%)$ \\
\hline &
\end{tabular}

(acetaminophen) were the most frequently used products within the study period. One cardiac arrest occurred with a successful use of an AED. One portable hyperbaric chamber was also used. In 112 patients, hut guardians contacted the nearest medical dispatch center for medical advice. In 32 (10\%) patients, the hut guardian was assisted by a nurse or a physician who was present in the hut as a customer. Helicopter evacuation was required in $64(20 \%)$ patients. According to these findings, we proposed a typical first aid kit that could be made available in each mountain hut (Table 3).

\section{Discussion}

In this prospective study, we described the actual first aid resources available in French mountain huts. The role of the hut guardian was considered valuable by all professionals involved in medical care in this context. We also conducted the first epidemiological study regarding MEs managed by hut guardians, demonstrating the low prevalence of these events. However, this study also 
Table 2. Characteristics of the 221 trauma-related injuries occurring in 126 French huts

\begin{tabular}{lr}
\hline Injury & $N(\%)$ \\
\hline Mechanism & \\
Fall & $45(20)$ \\
Work-related injury ${ }^{*}$ & $14(6)$ \\
Snow sliding & $11(5)$ \\
Climbing accident & $7(3)$ \\
Animal ${ }^{\dagger}$ /Insect bite & $10(5)$ \\
Stone/Ice fall & $6(3)$ \\
Horse accident & $4(2)$ \\
Type & \\
Skin cuts & $81(37)$ \\
Ankle twist & $35(16)$ \\
Feet blisters & $22(10)$ \\
Muscle/Tendon injuries & $16(7)$ \\
Animal bite & $9(4)$ \\
Fracture & $9(4)$ \\
Traumatic brain injury & $9(4)$ \\
Burns & $9(4)$ \\
Toenail injury & $5(2)$ \\
Back pain & $4(2)$ \\
Joint dislocation & $3(1)$ \\
Other & $15(7)$ \\
Location & \\
Lower extremity & $64(29)$ \\
Upper extremity & $9(4)$ \\
Torso & $9(4)$ \\
Multiple trauma & $9(4)$ \\
* Hand cuts or burns. & $4(4)$ \\
${ }^{\dagger}$ Snake bites (vipers only). & \\
& \\
\hline
\end{tabular}

highlighted the prominent role played by hut guardians in managing these situations. In light of these findings, we further proposed a first aid kit in each refuge.

In Europe, mountain huts geographically cover numerous areas with 430 huts recorded in Italy, 400 huts in the Alpenverein (South Tyrol, Austria, Germany), 200 huts in Switzerland, 207 huts in Bulgaria, 156 huts in Slovenia, 44 huts in Norway, and 28 huts in Spain (only in the Aragon region). ${ }^{4}$ Out of the 236 French huts, two thirds agreed to participate in the study. This high proportion of hut guardians willing to participate might reveal considerable concern for medical issues, although MEs rarely occurred. Indeed, 306 individuals presenting MEs accounted for less than $1 \%$ of the total population who spent a night in high-altitude huts within the study period. The low prevalence of these events was corroborated by an American observational study on outdoor activities. ${ }^{5}$ From a public health standpoint, our findings illustrated the key role of hut guardians in first aid. Conversely, from the hut guardian's standpoint, it is challenging to deal with rare medical issues. However, mountaineers were found to have poor knowledge regarding the main life-threatening emergencies occurring at high altitude. ${ }^{6}$ This finding further supported the valuable role played by hut guardians as first aid responders.

The present epidemiological study of MEs was conducted in France, and medical situations might differ across the world. Similar results were found with hikers on the Appalachian trail, ${ }^{7}$ although a higher proportion of trauma-related injury was reported in American mountaineers. ${ }^{8}$ The severity of the reported MEs in our case was low, with only $10 \%$ of total MEs considered to be severe from the clinical standpoint. The high availability of mountain rescue services might explain these results because severe cases were directly rescued by helicopter. ${ }^{9}$ These results supported the implementation of AEDs, as recommended by the international commission for mountain emergency medicine ICAR-MEDCOM, ${ }^{2}$ and increased risk of sudden death in mountain and highaltitude areas further confirmed this recommendation. $^{10-12}$

BLS training is required for hut guardians in certain European countries, but no such training is required in France. Nevertheless, many guardians were appropriately trained. Our interviews revealed that hut guardians expected to receive specific knowledge about first aid training. Training should extend beyond classic BLS or first aid courses and should take into account specialized wilderness medical training as recommended by the Wilderness Medicine Society. ${ }^{13}$ These recommendations include radio communication skills and the ability to safely assist with helicopter operations. Hut guardians have to be trained to contact rescue centers by telephone or radio and should be able to assist helicopter emergency missions. In bad weather or during the night, they should be able to provide advanced first aid using medication available in the first aid kit, assisted by telemedicine. ${ }^{14,15}$ They should be aware of acute mountain sickness symptoms, and finally they should be trained in providing educational activities regarding wilderness hazards. Considering the present results, we also proposed a standard composition for the first aid kit. As recommended by ICAR and the mountain hut initiative in Italy, ${ }^{16}$ a separate compartment should be reserved for health care professionals only (modular medical kit). However, if national regulations allow prescription by phone, emergency medications with possible side effects could be used under a physician's supervision. In France an emergency physician is always available by phone at the dispatching center.

We acknowledge several limitations to this study. First, the study was limited to the summer season when 


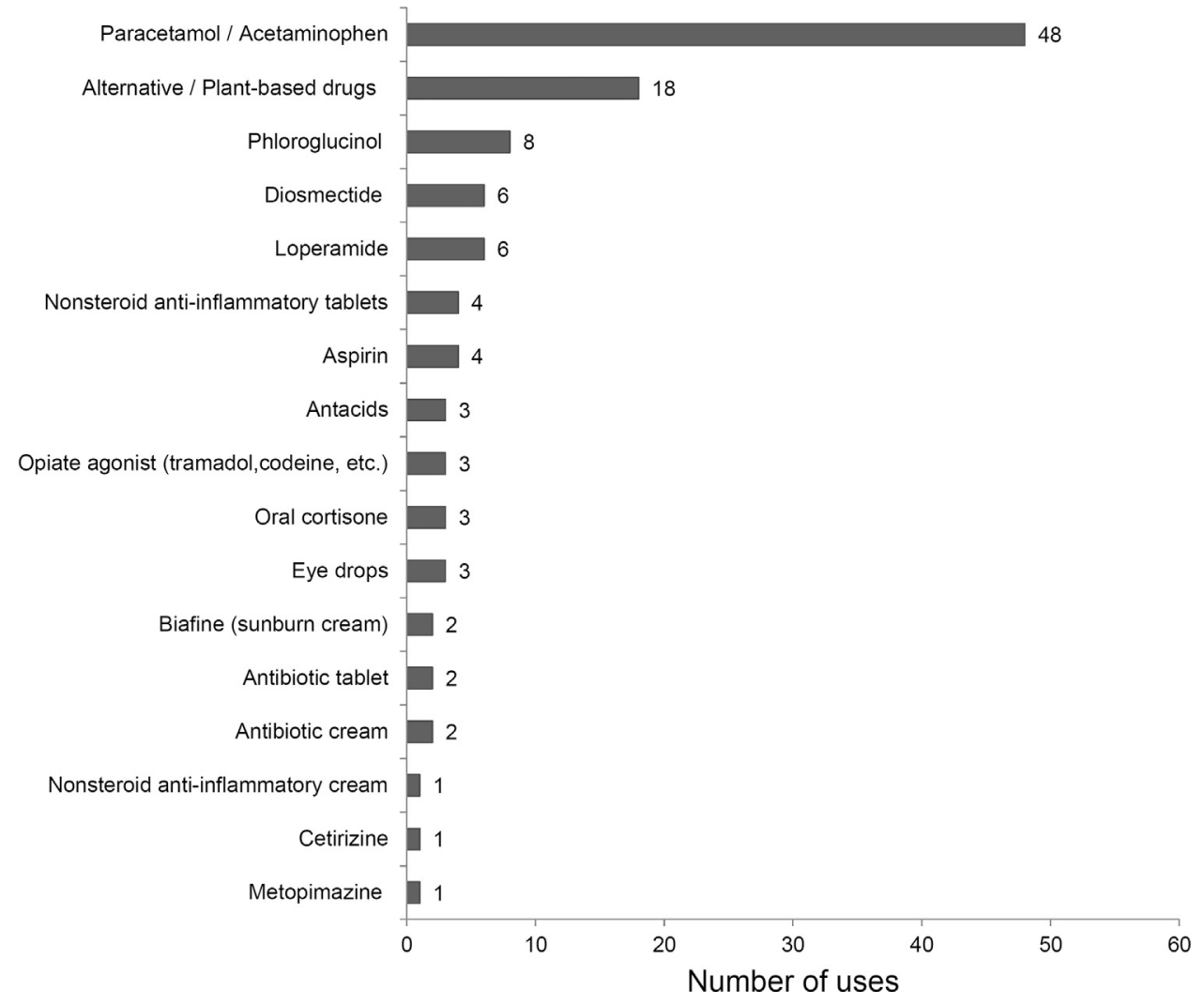

Figure 4. Medications used by hut guardians for the management of medical events within the study period.

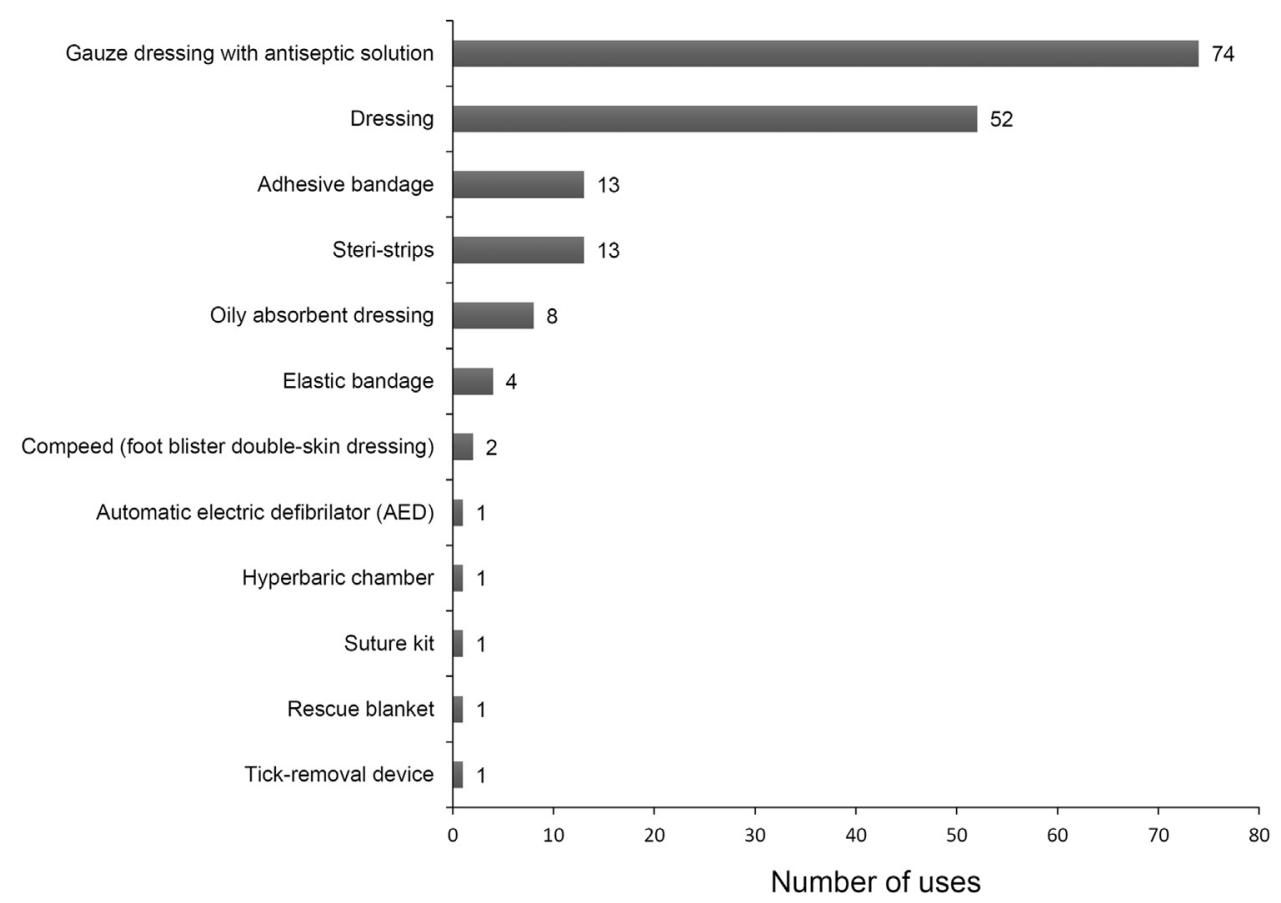

Figure 5. Devices used by hut guardians for the management of medical events occurring within the study period. 
Table 3. Proposal for first aid kit contents, considering the medical events observed

\begin{tabular}{|c|c|}
\hline Drugs & Symptoms \\
\hline \multicolumn{2}{|l|}{ All huts } \\
\hline Paracetamol/Acetaminophen & Pain and fever \\
\hline Tramadol, codeine & Severe pain \\
\hline $\begin{array}{l}\text { Ketoprofen (nonsteroidal } \\
\text { anti-inflammatory) }\end{array}$ & Moderate pain \\
\hline $\begin{array}{l}\text { Phloroglucinol } \\
\text { (antispasmodic) }\end{array}$ & Abdominal pain \\
\hline Racecadotril & Diarrhea \\
\hline Domperidone/Metopimazine & Nausea and vomiting \\
\hline Prednisolone & Allergy/Asthma \\
\hline Cetirizine & Allergy \\
\hline Esomeprazole/Pantoprazole & Stomach pain \\
\hline Picloxydine (eye drops) & Eye disease \\
\hline Physiologic serum & Eye disease \\
\hline Oxybuprocaine & Eye disease \\
\hline Benzodiazepine* & Anxiety \\
\hline Salbutamol ${ }^{*}$ & Asthma \\
\hline Nitro spray ${ }^{*}$ & Chest pain \\
\hline Acetylsalicylic acid ${ }^{*}$ & Chest pain \\
\hline \multicolumn{2}{|c|}{ Life-threatening emergencies only } \\
\hline IV epinephrine ${ }^{*}$ & Cardiac arrest \\
\hline IM epinephrine* (Anapen) & Anaphylactic shock \\
\hline IV morphine* & Severe pain \\
\hline IV Diazepam/Clonazepam* & Seizure \\
\hline Pocket mask & Cardiac arrest \\
\hline \multicolumn{2}{|l|}{ Huts $>3500 \mathrm{~m}$} \\
\hline Acetazolamide $^{*}$ & Acute mountain sickness \\
\hline Nifedipine* & Acute mountain sickness \\
\hline Hyperbaric chamber & Acute mountain sickness \\
\hline Oxygen bottle + mask & Acute mountain sickness \\
\hline \multicolumn{2}{|l|}{ Medical devices } \\
\hline \multicolumn{2}{|l|}{ Antiseptic solution } \\
\hline \multicolumn{2}{|l|}{ Dressing } \\
\hline \multicolumn{2}{|l|}{ Compress dressing } \\
\hline \multicolumn{2}{|l|}{ Steri-Strips } \\
\hline \multicolumn{2}{|l|}{ Adhesive bandage } \\
\hline \multicolumn{2}{|l|}{ Nonadhesive bandage } \\
\hline \multirow{2}{*}{\multicolumn{2}{|c|}{$\begin{array}{l}\text { Sam Split immobilization } \\
\text { device }\end{array}$}} \\
\hline & \\
\hline \multicolumn{2}{|l|}{ Wrap ice/Cold pack } \\
\hline \multicolumn{2}{|l|}{ Adhesive tape } \\
\hline \multicolumn{2}{|l|}{ Vaseline } \\
\hline \multicolumn{2}{|l|}{ Hydroalcoholic gel } \\
\hline \multicolumn{2}{|l|}{ Gloves } \\
\hline \multicolumn{2}{|l|}{ Survival blanket } \\
\hline \multicolumn{2}{|l|}{ Scissors/Tweezers } \\
\hline \multicolumn{2}{|l|}{ Tick removal device } \\
\hline Pocket mask & \\
\hline
\end{tabular}

\footnotetext{
* These drugs have to be located in a special compartment reserved for health care professionals only, and not used by hut guardians without a physician's agreement (according to national regulations).
}

hiking was the leading cause of medical or traumarelated pathologies. We cannot exclude that epidemiology would be different in other seasons. Second, even though this study was the largest database on this topic, the data collected were not exhaustive. For instance, we did not check for a simple correlation with evacuation records and hospital records to test the reliability of the data collected. Only motivated hut guardians agreed to participate in all phases of the study. However, with two thirds of all hut guardians participating, the sample size may be considered sufficient to extrapolate the findings to all mountaineers within the study period. Third, we combined qualitative and quantitative approaches. This method was essential to explore the prevalence of medial issues together with the role played by hut guardians in managing such situations. Qualitative methods are not commonly used but provide additional data beyond numbers and percentages. ${ }^{17-19}$ At the time of the study, this approach gave us important information regarding the expectations of hut guardians and professionals involved in mountain rescue. New concepts and ideas arose, which should be further explored in quantitative studies.

\section{Conclusions}

Few MEs occurred in the population frequenting mountain huts during the summer. However, each event was challenging for hut guardians as first aid responders. Hut guardians would benefit from specific training in wilderness BLS. We also proposed a standardized first aid kit that could be used in relation with a medical dispatching center through telemedicine. Its content should be adapted to specific epidemiology regarding the altitude and location of the corresponding mountain hut. Whether the composition of this first aid kit would be sufficient to face all medical situations remains to be seen.

Acknowledgments: The authors wish to thank all hut guardians who agreed to participate in the study, with particular thanks to Freddy Maignan (refuge du Promontoire, la Meije, France), president of the Mountain Wilderness Society in France and president of the Hut Guardian Syndicate. The authors also extend their thanks to the French Federation of Alpine Clubs (FFCAM), Dr Stephane Bare, president of PHAREFUGE, for his help in contacting hut guardians and Dr Audrey Faurite for her assistance in data collection.

Author Contributions: Study concept and design (MB, JCI, AC, PSG, AP, FC, GD, JLB, RB, PB); obtaining funding (MB, JCI, AC, PSG, AP, FC, GD, JLB, RB, PB); acquisition of data (MB, JLB, RB, $\mathrm{PB})$; drafting of the manuscript $(\mathrm{MB}, \mathrm{PB})$; critical revision of the manuscript (MB, JCI, AC, PSG, AP, FC, GD, JLB, RB, PB); and approval of final manuscript (MB, PB).

Financial/Material Support: None.

Disclosures: None. 


\section{Supplementary Materials}

Supplementary material cited in this article is available online at http://dx.doi.org/10.1016/j.wem.2016.08.002.

\section{References}

1. Vogt C, Forster H, Wiget U. Content of a mountain refuge's pharmacy. ICAR recommendation nr. 4. In: Elsensohn F, ed. Consensus Guidelines on Mountain Emergency Medicine and Risk Reduction. Zurich and Bern, Switzerland: International Commission for Alpine Rescue and International Mountaineering and Climbing Federation; 1996:33.

2. Elsensohn F, Agazzi G, Syme D, Swangard M, Facchetti $\mathrm{G}$, Brugger $\mathrm{H}$. The use of automated external defibrillators and public access defibrillators in the mountains: official guidelines of the international commission for mountain emergency medicine ICAR-MEDCOM. Wilderness Environ Med. 2006;17:64-66.

3. Guest G, Bunce A, Johnson L. How many interviews are enough? An experiment with data saturation and variability. Field Method. 2006;18:59-82.

4. ODIT-France. Les Refuges de Montagne en Europe: Approche Comparative sur 10 Pays de Differents Massifs (Alpes, Pyrénées, Balkans, Scandinavie). Paris: ODIT France; 2009.

5. Flores AH, Haileyesus T, Greenspan AI. National estimates of outdoor recreational injuries treated in emergency departments, United States, 2004-2005. Wilderness Environ Med. 2008;19:91-98.

6. Kuepper T, Wermelskirchen D, Beeker T, Reisten O, Waanders R. First aid knowledge of alpine mountaineers. Resuscitation. 2003;58:159-169.

7. Boulware DR, Forgey WW, Martin WJ 2nd. Medical risks of wilderness hiking. Am J Med. 2003;114:288-293.
8. Ela GK. Epidemiology of wilderness search and rescue in New Hampshire, 1999-2001. Wilderness Environ Med. 2004; 15:11-17.

9. Jacquot C, Mongenot F, Payen JF, et al. Severe trauma patients in a mountain area: an observational study [in French]. Ann Fr Anesth Reanim. 2011;30:730-733.

10. Burtscher M. Risk of cardiovascular events during mountain activities. Adv Exp Med Biol. 2007;618:1-11.

11. Burtscher M, Pachinger O, Schocke MF, Ulmer H. Risk factor profile for sudden cardiac death during mountain hiking. Int J Sports Med. 2007;28:621-624.

12. Burtscher M, Philadelphy M, Likar R. Sudden cardiac death during mountain hiking and downhill skiing. $N$ Engl J Med. 1993;329:1738-1739.

13. Johnson DE, Schimelpfenig T, Hubbell F, et al. Minimum guidelines and scope of practice for wilderness first aid. Wilderness Environ Med. 2013;24:456-462.

14. Ekeland AG, Bowes A, Flottorp S. Effectiveness of telemedicine: a systematic review of reviews. Int J Med Inform. 2010;79:736-771.

15. Conseil National de l'Ordre des Médecins. Télémédecine Les préconisations du Conseil National de l'Ordre des Médecins. Paris: Janvier; 2009.

16. Barcella L, Agazzi G, Malgrati D, et al. First aid and public access defibrillation in mountain huts: the Mountain Huts Initiative of the Bergamo section of the Club Alpino Italiano. Wilderness Environ Med. 2010;21:379-381.

17. Malterud K. The art and science of clinical knowledge: evidence beyond measures and numbers. Lancet. 2001; 358:397-400.

18. Malterud K. Qualitative research: standards, challenges, and guidelines. Lancet. 2001;358:483-488.

19. Pope C, Mays N. Reaching the parts other methods cannot reach: an introduction to qualitative methods in health and health services research. BMJ. 1995;311:42-45. 